\title{
Continuous live imaging of adult neural stem cell division and lineage progression in vitro
}

\author{
Marcos R. Costa ${ }^{1,2, *, \dagger}$, Felipe Ortega ${ }^{3, *}$, Monika S. Brill ${ }^{3}$, Ruth Beckervordersandforth ${ }^{4}$, Ciro Petrone ${ }^{4}$, \\ Timm Schroeder ${ }^{4}$, Magdalena Götz ${ }^{3,4,5}$ and Benedikt Berninger ${ }^{3,4, \dagger}$
}

\begin{abstract}
SUMMARY
Little is known about the intrinsic specification of adult neural stem cells (NSCs) and to what extent they depend on their local niche. To observe adult NSC division and lineage progression independent of their niche, we isolated cells from the adult mouse subependymal zone (SEZ) and cultured them at low density without growth factors. We demonstrate here that SEZ cells in this culture system are primarily neurogenic and that adult NSCs progress through stereotypic lineage trees consisting of asymmetric stem cell divisions, symmetric transit-amplifying divisions and final symmetric neurogenic divisions. Stem cells, identified by their astro/radial glial identity and their slow-dividing nature, were observed to generate asymmetrically and fast-dividing cells that maintained an astro/radial glia identity. These, in turn, gave rise to symmetrically and fast-dividing cells that lost glial hallmarks, but had not yet acquired neuronal features. The number of amplifying divisions was limited to a maximum of five in this system. Moreover, we found that cell growth correlated with the number of subsequent divisions of SEZ cells, with slow-dividing astro/radial glia exhibiting the most substantial growth prior to division. The fact that in the absence both of exogenously supplied growth factors and of signals provided by the local niche neurogenic lineage progression takes place in such stereotypic fashion, suggests that lineage progression is, to a significant degree, cell intrinsic or pre-programmed at the beginning of the lineage.
\end{abstract}

KEY WORDS: Asymmetric cell division, Astroglia, Neurogenesis, Radial glia, Subependymal zone, Time-lapse video microscopy, Mouse

\section{INTRODUCTION}

The adult olfactory bulb (OB) is constantly supplied with newly generated neurons (Zhao et al., 2008) that are derived from adult neural stem cells (NSCs) residing in distinct compartments of the wall of the lateral ventricle [subependymal zone (SEZ)] and the rostral migratory stream (RMS) (Alonso et al., 2008; Brill et al., 2009; Hack et al., 2005; Merkle et al., 2007). Despite active research on adult NSCs, major questions of stem cell biology remain unanswered. For example, are adult NSCs fully dependent on their local niche, or do niche-independent cell-intrinsic mechanisms contribute to the regulation of stem cell properties and lineage progression? Heterotypic transplantation of in vitro propagated adult SEZ stem and progenitor cells has demonstrated that these cells maintain their regional specification (Merkle et al., 2007), but nothing is known about the behaviour of adult NSCs with regard to their mode of cell division and lineage progression outside their niche. To address such questions, single cell or low density cultures have provided valuable information for NSCs isolated from the embryo (Qian et al., 1998; Qian et al., 2000; Ravin et al., 2008; Shen et al., 2006). Single-cell cultures of

\footnotetext{
${ }^{1}$ Instituto do Cérebro, Universidade Federal do Rio Grande do Norte (UFRN), 59072970 Natal, Brazil. ${ }^{2}$ Edmond and Lily Safra International Institute of Neuroscience of Natal (ELS-IINN), 59066-060 Natal, Brazil. 'Department of Physiological Genomics, Institute of Physiology, Ludwig-Maximilians University Munich, Schillerstrasse 46, D-80336 Munich, Germany. ${ }^{4}$ Institute for Stem Cell Research, National Research Center for Environment and Health, Ingolstädter Landstrasse 1, D-85764 Neuherberg, Germany. ${ }^{5}$ Munich Center for Integrated Protein Science CiPSM, Butenandtstraße 5-13, Munich 81377, Germany.

*These authors contributed equally to this work

${ }^{\dagger}$ Authors for correspondence (benedikt.berninger@|rz.uni-muenchen.de; mrcosta@neuro.ufrn.br)
}

Accepted 29 December 2010 cortical stem and progenitor cells have revealed a strikingly cellautonomous programme of lineage progression underlying the sequential generation of distinct types of cortical principal neurons (Shen et al., 2006) and astroglia (Qian et al., 2000). So far, no comparable single-cell analysis has been performed for adult NSCs, which differ substantially from embryonic NSCs, for example by their much slower cell cycle. Cell-intrinsic contributions to adult NSC regulation can be disentangled only by isolation from their niche, but so far adult NSCs have only been cultivated and propagated either in high growth factor conditions (Laywell et al., 2000; Reynolds and Weiss, 1992) or on astrocyte feeder layers (Lim and Alvarez-Buylla, 1999).

Another unresolved question is whether adult NSCs self-renew by asymmetric cell division or by different population-based mechanisms. Here, we refer to asymmetric cell division as the acquisition of distinct cell fates by the two daughter cells. Adult NSC might divide asymmetrically giving rise to a renewed NSC while at the same time also producing lineage-restricted progeny or, alternatively, might divide symmetrically with both daughter cells either retaining stem cell identity or undergoing differentiation, a decision regulated either by asymmetrically inherited intrinsic determinants, stochastically or by niche-derived extrinsic signals (Shroeder, 2008). The classical in vitro test for stem cell self-renewal, the neurosphere assay (Reynolds and Rietze, 2005), does not allow the question of the mode of NSC division to be addressed owing to the high cell density. Besides, the assay depends on the exposure of NSCs to mitogenic growth factors, which have confounding effects on cell fate decisions (Doetsch et al., 2002). Of similar importance is the question of to what extent different SEZ lineages involve an intermediate, transit-amplifying progenitor (TAP) state and whether proliferation of these intermediates depends on the local niche. A general model for adult neurogenesis proposes a series of transitions from slow-dividing astro/radial glial NSCs, that is cells with both 
astroglial and radial glial characteristics (also known as type B cells), via TAPs (type $\mathrm{C}$ cells) to neuroblasts (type A cells) (Kriegstein and Alvarez-Buylla, 2009). However, this model is based on population analysis in vivo, and virtually nothing is known about the actual enactment of the mode of cell division, lineage transition and generation of intermediate amplifying populations.

As answering the above questions requires continuous observation of single cells in isolation by live imaging (Schroeder, 2008), we have exploited a recently developed preparation of the adult SEZ (Brill et al., 2008; Brill et al.,2009) in which cells are cultivated under adherent conditions in the absence of growth factors or an astrocyte feeder layer (Lim and Alvarez-Buylla, 1999), in isolation from their niche. This system enabled us to continuously follow dividing stem and progenitor cells from the adult SEZ by single cell tracking over about a week to study the cell-intrinsic processes underlying the transition from adult NSCs to neurons.

\section{MATERIALS AND METHODS Cell culture}

SEZ cultures were prepared from the lateral wall of the lateral ventricle of young adult (8-12 weeks) C57/B16 mice (Johansson et al., 1999). Briefly, tissue was enzymatically dissociated in $0.7 \mathrm{mg} / \mathrm{ml}$ hyaluronic acid (SigmaAldrich) and $1.33 \mathrm{mg} / \mathrm{ml}$ trypsin (Sigma-Aldrich) in Hanks' Balanced Salt Solution (HBSS; Invitrogen) with $2 \mathrm{mM}$ glucose at $37^{\circ} \mathrm{C}$ for 30 minutes. Dissociation was stopped by adding an equal volume of an ice-cold medium consisting of $4 \%$ bovine serum albumin (BSA; Sigma-Aldrich) in Earle's Balanced Salt Solution (EBSS; Invitrogen) buffered with 20 mM HEPES (Invitrogen). Cells were then centrifuged at $200 \mathrm{~g}$ for 5 minutes, resuspended in ice-cold medium consisting of $0.9 \mathrm{M}$ sucrose (Sigma-Aldrich) in $0.5 \times \mathrm{HBSS}$, and centrifuged for 10 minutes at $750 \mathrm{~g}$. The cell pellet was re-suspended in $2 \mathrm{ml}$ ice-cold medium consisting of 4\% BSA in EBSS buffered with $2 \mathrm{mM}$ HEPES, and the cell suspension was placed on top of $12 \mathrm{ml}$ of the same medium and centrifuged for 7 minutes at $200 \mathrm{~g}$. The cell pellet was finally re-suspended in DMEM/F12 Glutamax (Invitrogen) supplemented with B27 (Invitrogen), 2 mM glutamine (Sigma), 100 units $/ \mathrm{ml}$ penicillin (Invitrogen), $100 \mu \mathrm{g} / \mathrm{ml}$ streptomycin (Invitrogen), buffered with $8 \mathrm{mM}$ HEPES, and cells were plated on poly-D-lysine (Sigma) coated coverslips at a density of 200-300 cells per $\mathrm{mm}^{2}$. Four hours later, cells were transduced with pseudotyped retroviruses using the CMMP construct encoding green fluorescent protein (GFP) (Hack et al., 2004).

\section{Immunocytochemistry and clonal analysis}

Cultures were fixed in 4\% paraformaldehyde (PFA) in phosphate-buffered saline (PBS) for 15 minutes at room temperature and processed for antibody staining as described previously (Costa et al., 2008). Antibodies used were: mouse IgG2b anti- $\beta I I I$ tubulin (Sigma-Aldrich; 1:1000), guinea pig anti-DCX (Chemicon; 1:500), rabbit anti-GFAP (DakoCytomation; 1:500), chicken anti-GFP (Aves Labs, OR, USA; 1:2000), mouse IgG1 anti-MAP2 (Sigma-Aldrich; 1:1000), mouse IgM anti-O4 (Dr J. Price, Institute of Psychiatry, London, UK; 1:200) and rabbit anti-olig2 (Millipore; 1:500). After 7 days, in vitro individual clones were identified on the basis of GFP immunoreactivity and classified according to their marker expression as (1) neuronal (all cells $\beta$-III tubulin-positive), (2) glial (all cells either O4- or GFAP-positive) or (3) mixed (containing both $\beta$-III tubulin- and GFAP-positive cells).

\section{Time-lapse video microscopy}

Time-lapse video microscopy (Costa et al., 2008; Eilken et al., 2009) of primary SEZ cultures was performed with a cell observer (Zeiss) at constant conditions of $37^{\circ} \mathrm{C}$ and $8 \% \mathrm{CO}_{2}$. Phase contrast images were acquired every 2-4 minutes for 6-8 days using a $20 \times$ phase contrast objective (Zeiss) and an AxioCamHRm camera with a self-written VBA module remote controlling Zeiss AxioVision 4.7 software (Rieger and Schroeder, 2009). Single-cell tracking was performed using a self-written computer program (TTT) (Rieger and Schroeder, 2009). Movies were assembled using ImageJ 1.42q (National Institute of Health, MD, USA) software and are played at a speed of three frames per second.

\section{Cell size quantification}

For cell area analysis, images recorded by time-lapse video microscopy were imported as an image sequence using the software ImageJ $1.42 \mathrm{q}$. Cells were classified as slow-dividing or fast-dividing astroglia [based on mRFP1 (monomeric red fluorescent protein 1) (Hirrlinger et al., 2005) expression driven by the hGFAP promoter and cell cycle length $>$ or $<36$ hours, respectively], TAPs (dividing one, two or three rounds, losing RFP expression) and, finally, neuroblasts in order to assign the corresponding sizes to each class. As an approximation of cell size we measured the area covered by the soma and processes of each cell in $\mu \mathrm{m}^{2}$ during lineage progression (see also Fig. S1 in the supplementary material). Cell size was measured every 6 hours and 1 hour prior to division (maximum size) and 10 minutes after each cell division (minimum size). In order to confirm that measurement of cell area using this simple method accurately reflects cell size, we determined the diameter of cells just prior to division when cells typically adopt a spherical geometry (see Fig. S2A in the supplementary material). To confirm that cells that appear roundish do indeed have a spherical geometry, we performed confocal analysis of RFP-expressing cells followed by $3 \mathrm{D}$-reconstruction (see Fig. $\mathrm{S} \mathrm{A}^{\prime}$ in the supplementary material). By measuring the diameter of cells one frame (5 minutes) prior to division (see Fig. S2B in the supplementary material) we calculated the corresponding absolute cell area (see Fig. S2C in the supplementary material). Despite inherent differences in these measurements (absolute surface area versus visible area), there is an excellent correlation between these two measures (see Fig. S2E in the supplementary material). Although the absolute surface area is a more accurate measure, the area seen in the image can be more easily analysed using ImageJ software at the phases when cells do not exhibit a spherical geometry and gives useful information regarding the relative changes in cell size. Thus, the latter method was used when analysing cell growth over time along lineage progression. For quantification of the cell size at the moment prior to cell division we measured the cell diameter.

\section{Fluorescence-activated cell sorting (FACS)}

For FACS analysis we used 8-week-old hGFAP-GFP mice (Nolte et al., 2001) crossed to DCX-DsRed mice (Couillard-Despres et al., 2006), or wild-type mice (three independent experiments with one to five animals per experiment). After dissociation, SEZ cells were resuspended in PBS containing primary antibodies [allophycocyanin (APC)-conjugated antiCD133 (eBioscience; 1:100), biotinylated anti-EGF complexed with Alexa Fluor 647-streptavidin (Molecular Probes; 1:100) or isotype-matched control antibodies] and incubated for 20 minutes. Next, cells were incubated with propidium iodide (PI; 1:1000) for 10 minutes and analysed using a FACS Aria (BD). For further details see Beckervordersandforth et al. (Beckervordersandforth et al., 2010) (see Fig. S3 in the supplementary material)

\section{Electrophysiology}

Perforated patch-clamp recordings were performed as previously described (Heinrich et al., 2010).

\section{Statistical analysis}

Statistical tests were performed using GraphPad Prism 4.03 or MatLab 7.11; $P$ values were calculated by Mann-Whitney test, ANOVA with Tukey's post-hoc test or Kolmogorov-Smirnov two-sample test.

\section{RESULTS \\ Adult SEZ precursors proliferate in the absence of growth factors generating predominantly neuronal progeny \\ Cells were dissociated from the adult murine SEZ, plated onto poly-} D-lysine coated coverslips at a density of $200-300 \mathrm{cells} / \mathrm{mm}^{2}$ and maintained in chemically defined serum-free medium, without the addition of any mitogenic growth factors. Four hours after plating, cell identity was determined by immunocytochemistry for glial fibrillary acidic protein (GFAP), which labels astroglia (21.7\%; three independent experiments); doublecortin (DCX), which labels 

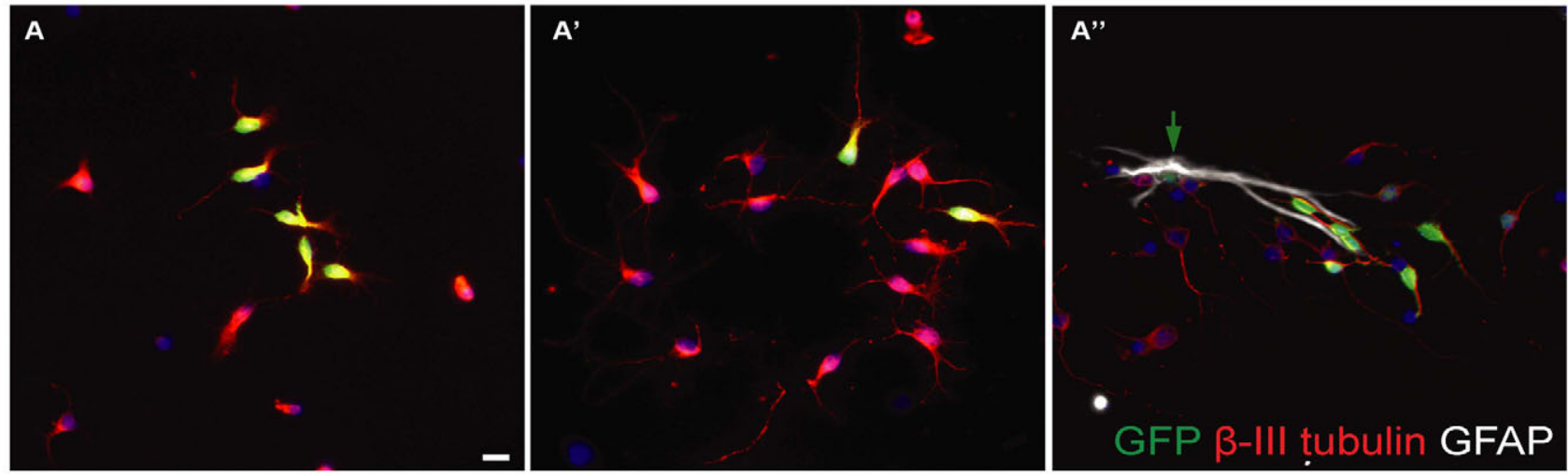

Retrovirus 7div

B

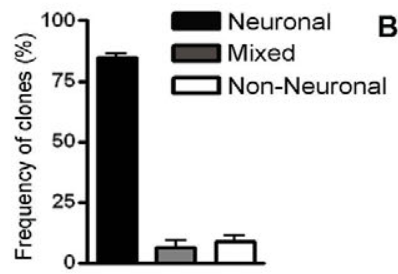

Video microscopy 7div

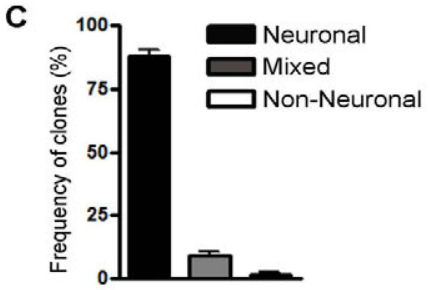

$B^{\prime}$

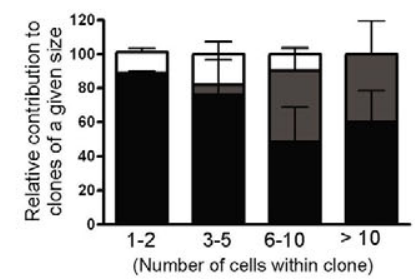

C'

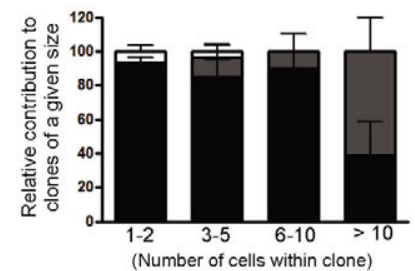

B"

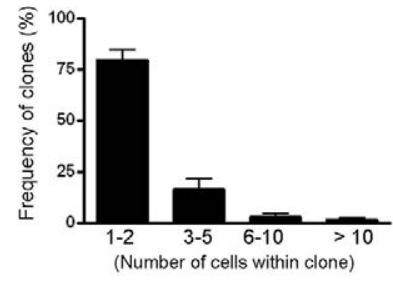

C"

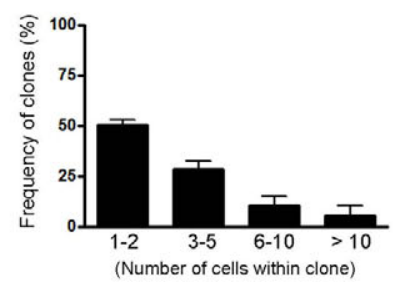

D

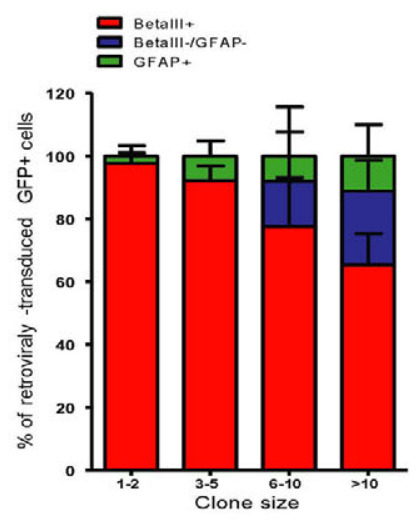

Fig. 1. Adult SEZ progenitor cells generate predominantly neuronal progeny. $\left(\mathbf{A}, \mathbf{A}^{\prime}\right)$ Clones derived from single progenitors transduced with a GFP-encoding retrovirus (green, GFP) containing only neurons (red, $\beta$-III tubulin) after 7 days in vitro (div). ( $\mathbf{A}^{\prime \prime}$ ) Clone containing both neurons and astrocytes (white, GFAP). Arrow indicates astroglial cell. (B) The overall frequency of different types of clones (neuronal, mixed, non-neuronal). $\left(\mathbf{B}^{\prime}\right)$ Relative contribution of neuronal, mixed and non-neuronal clones (see B for key) to clones of a given size (classified according to the number of cells contained within each clone). (B') Overall frequency of clones of different size ( $n=255$ clones; four independent experiments). (C) Overall frequency

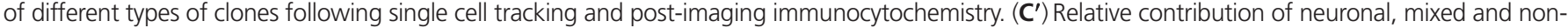
neuronal clones (see $\mathbf{C}$ for key) to clones of different size as revealed by single cell tracking. ( $\mathbf{C}^{\prime \prime}$ ) Frequency of clones of different size as revealed by single cell tracking. (D) Relative proportion of neurons (Betalll+; red), astroglia (GFAP+; green) and $\beta$-III tubulin/GFAP-double negative cells (Betalll-/GFAP-; blue) within clones of different size as revealed by clonal analysis. Error bars indicate s.e.m. Scale bar: $10 \mu \mathrm{m}$. SEZ, subependymal zone.

young neurons (68.8\%); and Olig2, which labels transit-amplifying precursors (TAPs; 9.5\%) (Doetsch et al., 2002), thus reflecting the relative proportions of these cells types in the adult SEZ in vivo (Colak et al., 2008) (see Fig. S4 in the supplementary material). To assess to what extent adult SEZ cells continue proliferating under these conditions and to follow the progeny of single proliferating cells, we infected cultures with a low titre of retroviral vectors encoding green fluorescent protein (GFP) that only becomes incorporated into the genome of the infected cells when they are undergoing cell division. By 2 days post infection (dpi), small clusters of GFP-positive cells could already be observed (see Fig. $\mathrm{S} 5 \mathrm{~A}$ in the supplementary material). These GFP-positive clusters increased in size with many cells subsequently acquiring a neuroblast-like morphology and the electrical properties of neurons (see Fig. S5A,B in the supplementary material). After 7 days in vitro (div) the majority of GFP-expressing cells were positive for $\beta$-III tubulin $(83 \pm 7 \%)$, with the remainder being GFAP $+(7 \pm 4 \%)$ or
O4+ $(<1 \%)$, or cells that had not yet acquired any marker for differentiated cell types $(9 \pm 6 \%)$; the latter are likely to be undifferentiated progenitors. Consistent with the predominance of neurogenesis in this culture system, the vast majority of clones (total number of clones analysed=255) were composed exclusively of neurons with the remaining clones being either mixed (containing non-neuronal cells alongside neurons) or exclusively glial (Fig. 1A-A",B; see Fig. S6 in the supplementary material). Of note, mixed clones were typically large, comprising mostly neurons and few astrocytes (Fig. 1A", $\mathrm{B}^{\prime}, \mathrm{D}$ ); the frequency of occurrence of the latter was considerably lower than the frequency of occurrence of small clones (Fig. 1B"). Glial clones were also rare and usually small (Fig. 1B) and very few (3 out of 255) contained oligodendroglial progeny (see Fig. S6 in the supplementary material). Notably, analysis of the clone composition following single cell tracking (see below) corroborated the data obtained by clonal analysis (Fig. 1C-C"). 

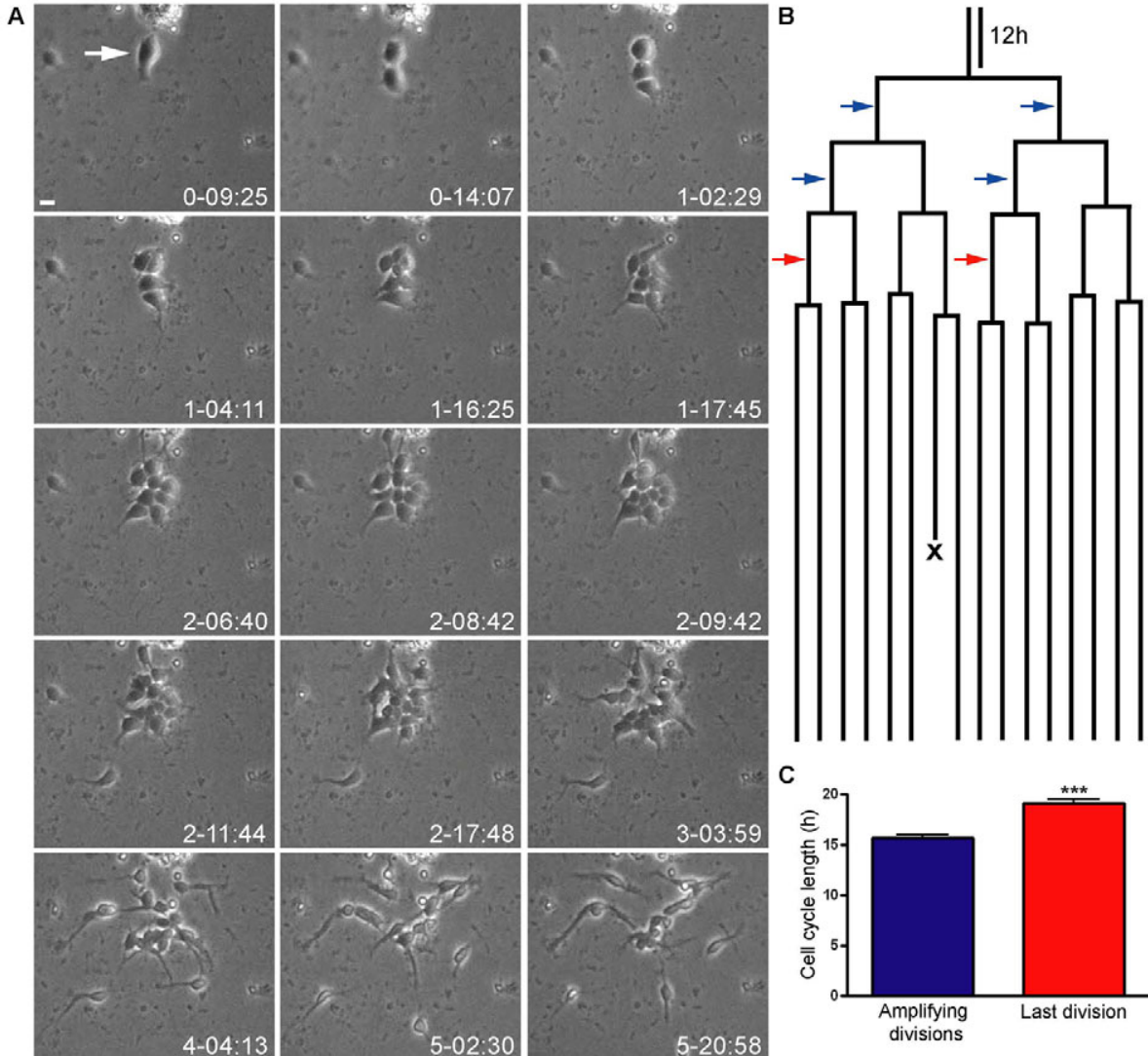

\section{C}

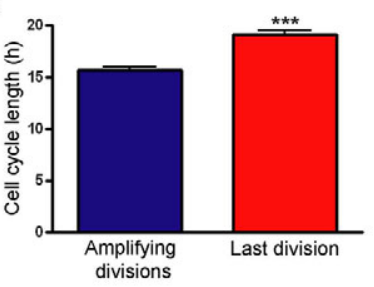

Fig. 2. Symmetric divisions give rise to transit-amplifying progenitors and neuroblasts. (A) Phase contrast images obtained by video time-lapse microscopy at different time points (day-hour:minute) show a fast-dividing cell (arrow) undergoing several rounds of symmetric division. Note the characteristic neuroblast morphology of the postmitotic cells observed after 3 days. At that stage, these cells also present a migratory behaviour similar to neuronal chain migration (see also Movie 1 in the supplementary material). (B) Lineage tree beginning with the cell shown in A (white arrow). Blue arrows indicate amplifying divisions, red arrows indicate last division. $X$ indicates cell death. (C) The average cell cycle length of fast-dividing cells that generate two proliferative cells (amplifying divisions, blue arrows in B) or two postmitotic cells (last division, red arrows in B). Error bars indicate s.e.m. ${ }^{* * *} P<0.001$, Mann-Whitney test. Scale bar: $10 \mu \mathrm{m}$.
Most neurons generated in these cultures acquired a GABAergic phenotype, consistent with the predominance of DLX (distal-less homeobox)-expressing progenitors in these cultures (Brill et al., 2008). Accordingly, in cultures prepared from GAD67::GFP mice (Tamamaki et al., 2003), most of the neuronal progeny in these cultures became GFP-positive ( $>95 \%$; see Fig. S7A,C in the supplementary material). Moreover, patch-clamp analysis demonstrated the vastly prevailing generation of functional GABAergic synapses (see Fig. S7 in the supplementary material). We could also observe a minority of glutamatergic neuron progenitors in these cultures, which we recently uncovered also in vivo (Brill et al., 2009).

\section{Continuous imaging reveals the presence of TAPs and their transition to neuroblasts}

Given the appropriate neurogenic fate of cells in this culture system, we then proceeded to single cell tracking by video time-lapse microscopy (Eilken et al., 2009; Shen et al., 2006). Single cell tracking was performed over periods of up to 8 days with images taken at 2-4 minute intervals in order to follow the fate of individual cells and their progeny (Costa et al., 2008; Eilken et al., 2009; Rieger and Schroeder, 2009). In numerous cases (89 out of 192 lineage trees analysed), SEZ cells were found to undergo symmetric proliferative divisions, characterised by a relatively fast cell cycle $(15.7 \pm 0.25$ hours, number of individual divisions analysed=88), with both daughter cells re-entering cell cycle (Figs 2, 3; see Movie 1 in the supplementary material). Lineage tree analysis revealed a striking degree of synchrony in the timing of the cell cycle between cellular siblings (Figs 2, 3). Conspicuously, with each cell cycle the soma size of the daughter cells appeared to be reduced compared with the mother cell (Fig. 2A; see also below). After one to four rounds of symmetric proliferative cell division, this pattern was superseded by a symmetric terminal division that resulted in the generation of two neuroblasts that began to extend neurites, exhibited migratory behaviour and were characterised by DCX expression (Fig. 2A,B, Fig. 3; see Movie 1 in the supplementary material). Cell cycle length prior to this terminal division was significantly longer than in the symmetric proliferative divisions $(19.2 \pm 0.42$, number of individual terminal divisions analysed $=78, P<0.001$ determined by MannWhitney test) (Fig. 2C).

In addition to the lineages described above, we also observed many instances (87 out of 192) of cells undergoing a terminal division without prior divisions (Fig. 3A), presumably representing progenitors that had been isolated at the final stage of lineage progression in vivo. Figure 3 shows a summary of various lineage trees that differ chiefly by the number of rounds of symmetric cell divisions prior to the neuroblast-producing terminal division, but otherwise exhibit a fairly similar pattern. We suggest that these trees represent cells isolated at different stages in their lineage progression. These patterns of symmetric proliferation correspond well to a model in which TAPs divide symmetrically for a limited number of cell divisions. The maximum number of symmetric cell divisions, comprising both proliferative and terminal ones, that we could observe was five (Fig. 3F,G). The frequency of cells undergoing one, two, three, four or five rounds of division diminished roughly exponentially (Fig. 3I).

\section{Direct imaging of asymmetric divisions within the astroglial NSC lineage}

Although most cells were found to exhibit a fast cell cycle, some cells underwent cell division after considerably longer time periods (52.9 \pm 3.2 hours, number of individual slow divisions 


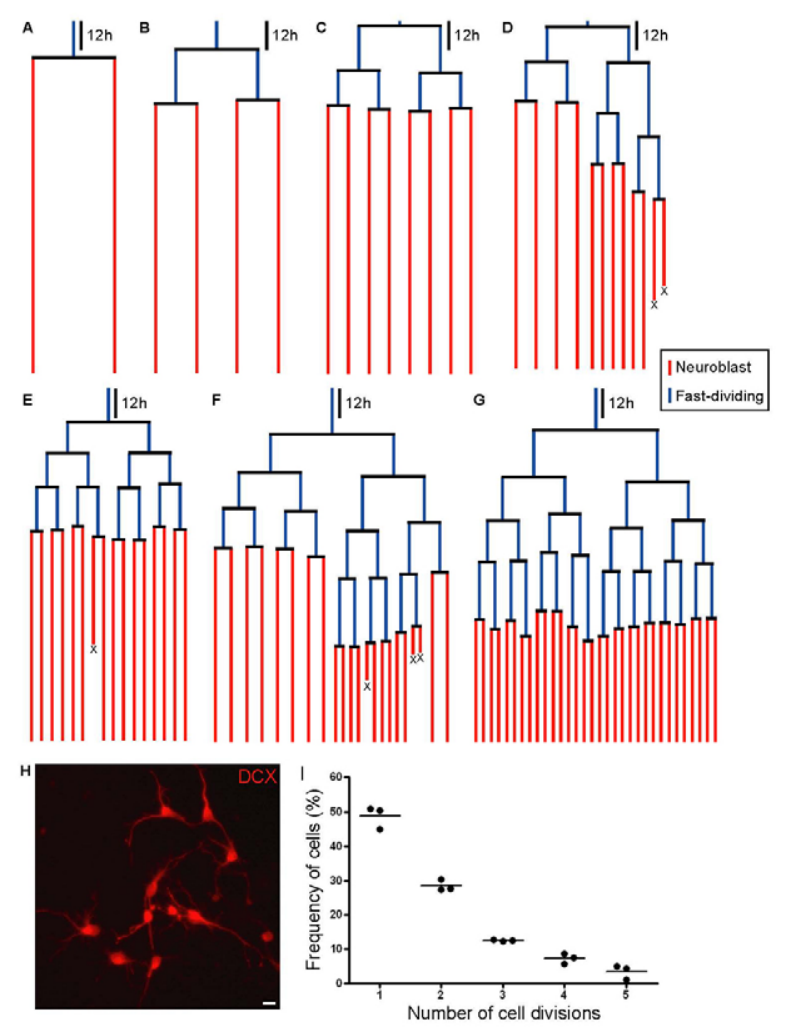

Fig. 3. Overview of distinct symmetric lineage trees. (A) Lineage tree obtained from a precursor giving rise to two postmitotic neuroblasts ( $n=87$ ). (B-G) More complex patterns of symmetric cell division with fast-dividing cells dividing symmetrically twice $(B, n=51)$, thrice ( $C ; n=22)$, four times ( $D-F ; n=12)$ and eventually five times $(F, G$; $n=5$ ) before finally giving rise to neuroblasts. $X$ indicates cell death. (H) Neuroblast identity of postmitotic progeny indicated by DCX immunoreactivity. Example of DCX-positive cells generated by the lineage tree shown in D. (I) The relative frequency of fast-dividing cells per independent experiment undergoing one to five rounds of cell division. Horizontal lines indicate group mean. Scale bar: $10 \mu \mathrm{m}$.

analysed=16). Most notably, the clones generated from such slowdividing cells were characterised by at least one asymmetric cell division. Figure 4 illustrates an example in which a slow-dividing cell, prior to the first division, grew considerably in size exhibiting large bipolar-oriented processes suggestive of an astro/radial glia identity (see also Movie 2 in the supplementary material). Subsequently, this cell generated two cells of similar morphology, but of a faster cell cycle, which in turn gave rise to two distinct branches: (1) a symmetrical branch (Fig. 4, blue arrow), virtually identical to the lineage trees described in the section above consisting of fast-dividing TAPs; and (2) an asymmetric branch (Fig. 4, black arrow). In addition to TAPs, this latter branch also maintained throughout all divisions a cell exhibiting astro/radial glial cell morphology similar to the founder cell, clearly distinct from the other fast-dividing cells. Notably, after a further round of division this astro/radial glial daughter cell carried out no further divisions throughout the remaining imaging time (Fig. 4, green arrow; see Movie 2 in the supplementary material).

This pattern of asymmetric lineage progression was found to be highly characteristic of slow-dividing cells (Figs 4, 5, 6). Postimaging immunocytochemistry confirmed the presence of one to a few GFAP-positive astroglia within this type of clone (Fig. 6; see
Figs S8, S9 in the supplementary material), whereas all other progeny was either positive for DCX, indicative of neurogenesis (Fig. 6; see Fig. S9 in the supplementary material), or markernegative, characteristic of TAPs (Fig. 5). The founder cells of these asymmetric clones are apparently characterised by the stem cell hallmark of self-renewal with an initiating slow-dividing astro/radial glial cell (Kriegstein and Alvarez-Buylla, 2009) maintaining throughout the entire lineage progression one to a few progeny of similar astro/radial glial identity, while at the same time initiating the stereotypic symmetric neurogenic lineages described above (Figs 4, 5, 6).

To corroborate the glial nature of these clonal founder cells, we continuously imaged SEZ cultures prepared from hGFAPRFP mice (Hirrlinger et al., 2005) in which adult SEZ stem cells have been found to be reporter-positive (Colak et al., 2008). Figure 6 shows two examples of slow-dividing cells upregulating RFP expression prior to cell division and generating clones comprising both neuronal and astroglial progeny. These data unambiguously show that slow-dividing cells are astro/radial glia that can give rise to mixed progeny. Consistent with a subsequent change in fate, reporter-fluorescence decreased rapidly in most of the progeny (Fig. 6). It is noteworthy that we also observed that the RFP expression driven by the hGFAP promoter was much reduced in astroglial cells generated from RFP-expressing astroglia (Fig. 6; see Fig. S10 in the supplementary material), indicating that high levels of RFP expression might be associated with the transition from a quiescent or slow-dividing state to entry into the cell cycle.

Although slow-dividing astroglia were typically at the base of lineage trees exhibiting asymmetric cell division, in no instance $(n=16)$ were these cells themselves found to divide asymmetrically, but rather they gave rise to two or four fast-dividing RFP-positive cells of astroglial morphology (Figs 5, 6; see Movie 3 in the supplementary material), indicating that the immediate progeny still retains an astro/radial glial identity. Indeed, post-imaging immunostaining of progeny at the four-cell stage of clones derived from slow-dividing cells exhibited GFAP immunoreactivity, confirming the astro/radial glial identity (see Fig. S11 in the supplementary material). Surprisingly, in all cases it was these faster dividing astro/radial glia that underwent asymmetric cell division (Fig. 5).

\section{Cell growth and size correlate with the specific stage of a cell within the lineage}

As noted above, within a lineage we observed a progressive decrease in soma size with each round of cell division. Prior to cell division, slow-dividing astro/radial glia underwent marked growth (see Fig. 4). In order to assess whether cell growth and size do indeed correlate with the specific stage of a cell within the lineage, we quantified the area of each cell (comprising both soma and processes) within a given clone every 6 hours as well as 1 hour before and 10 minutes after cell division (Fig. 7; see Fig. S1 in the supplementary material). Consistent with qualitative observations, we detected an increase in cell size from the time of the last division to the time just before the next, and this apparent growth was most conspicuous in the case of the slow-dividing founder cells of each clone (Fig. 7A,B). Interestingly, for the latter the growth rate appeared to accelerate before division (Fig. 7B). Moreover, despite an increase in cell size between one division and the next, the average maximum size of cells within a clone was found to diminish with each division throughout the progression from the slow-dividing 

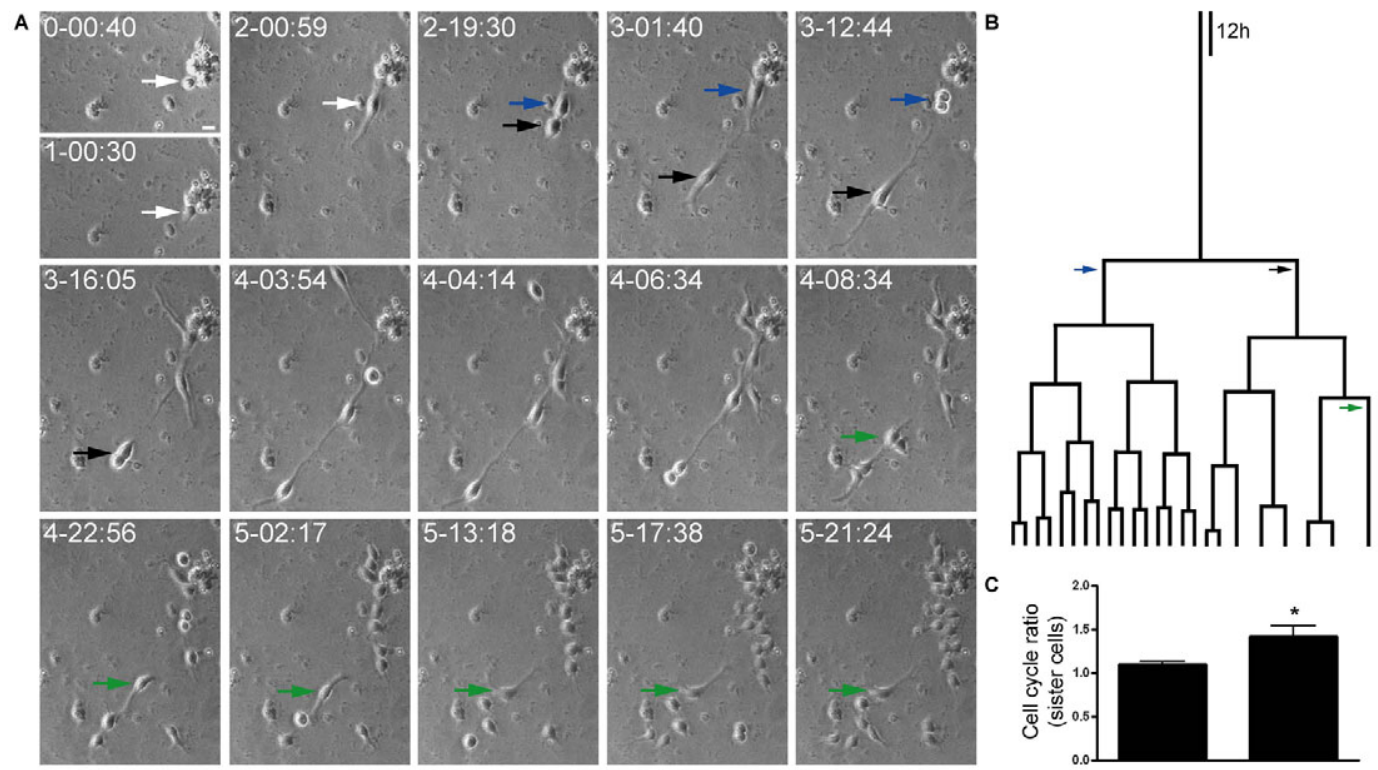

Fig. 4. Slow-dividing NSCs generate asymmetric lineage trees. (A) Phase contrast images obtained by time-lapse microscopy at different time points (day-hour:minute) show a slow-dividing cell that underwent the first round of cell division after nearly 3 days of imaging (white arrow) producing thereafter fast-dividing progeny. Note that whereas one daughter cell gave rise to a highly symmetric branch (symmetric cell divisions and synchronised cell cycles, blue arrows), the second daughter cell generated an asymmetric branch (asymmetric cell divisions and non-synchronised cell cycles, black arrows) that eventually gave rise to a non-dividing cell of a morphology similar to the founder cell (green arrows). (B) Lineage tree derived from the slow-dividing cell depicted in A. (C) The difference in the ratio between cell cycle lengths of sister cells in the symmetric (blue arrow) and asymmetric (black arrow) branches. Error bars indicate s.e.m. ${ }^{*} P=0.0272$, Mann-Whitney test. Scale bar: $10 \mu \mathrm{m}$. NSC, neural stem cell.

astro/radial glia to the neuroblast stage (Fig. 7B,C), with the conspicuous exception of cells that maintained astro/radial glial identity (Fig. 7A,B). As can be appreciated from Fig. 7A,B, such cells (see cell \#13) were found to differ markedly from their rather homogeneous cellular siblings with regard to size and growth, resembling in their growth pattern the original slowdividing founder cell of the clone. Thus, these data indicate that absolute cell size and overall growth correlate with the stage of the cell within the lineage, with slow-dividing astro/radial glia not only being the largest but also undergoing the most substantial growth, whereas fast-dividing astro/radial glia and TAPs get progressively smaller with each round of cell division.

In order to assess whether a similar decrease in cell size can also be observed during lineage progression from stem cell to neuroblasts in vivo, cells from the adult SEZ were freshly isolated and subjected to fluorescence-activated cell sorting (FACS) using distinct markers to distinguish astroglial stem cells (GFP transgene expression driven from a human GFAP promoter in

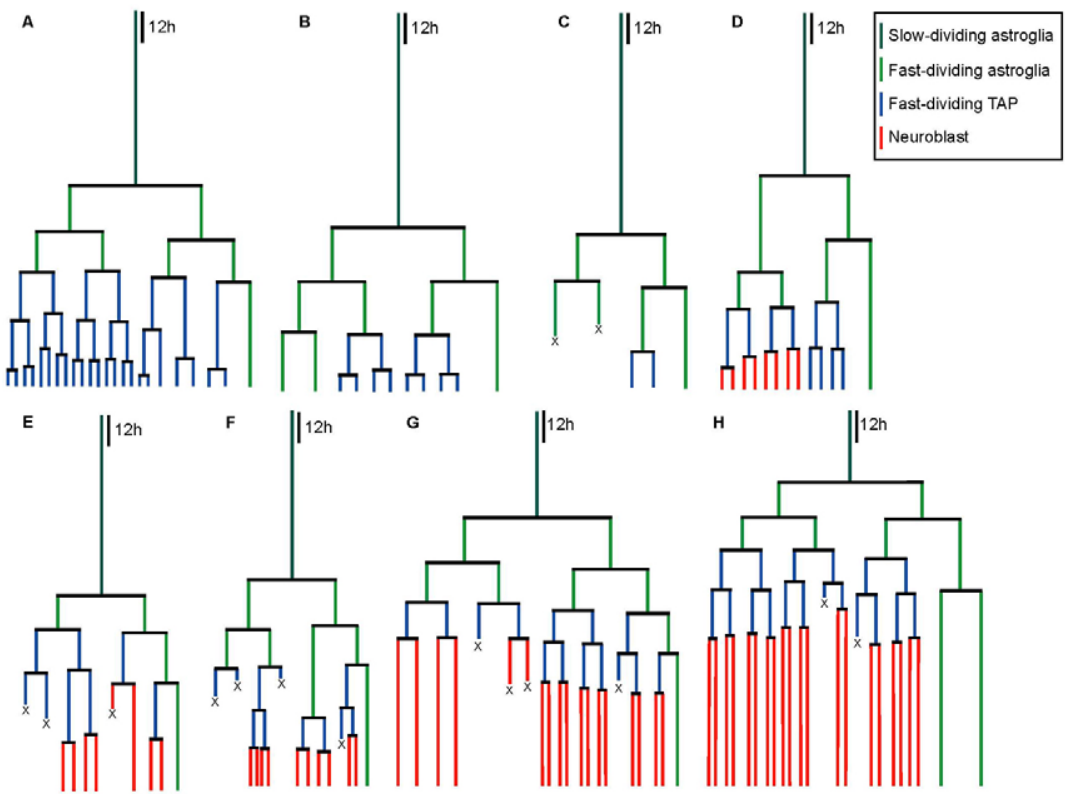

Fig. 5. Lineage trees generated by slow-dividing astro/radial glia. (A-H) Slow-dividing astro/radial glia (dark green lines; cell cycle length $\geq 36$ hours) typically generate two to four fast-dividing astro/radial glia (light green) which either divide symmetrically giving rise to fast-dividing TAPs (blue) or asymmetrically generating TAPs and astroglia. Note that cells identified as TAPs always generate neuroblasts (red). $X$ indicates cell death. TAP, transit-amplifying progenitor. 
A
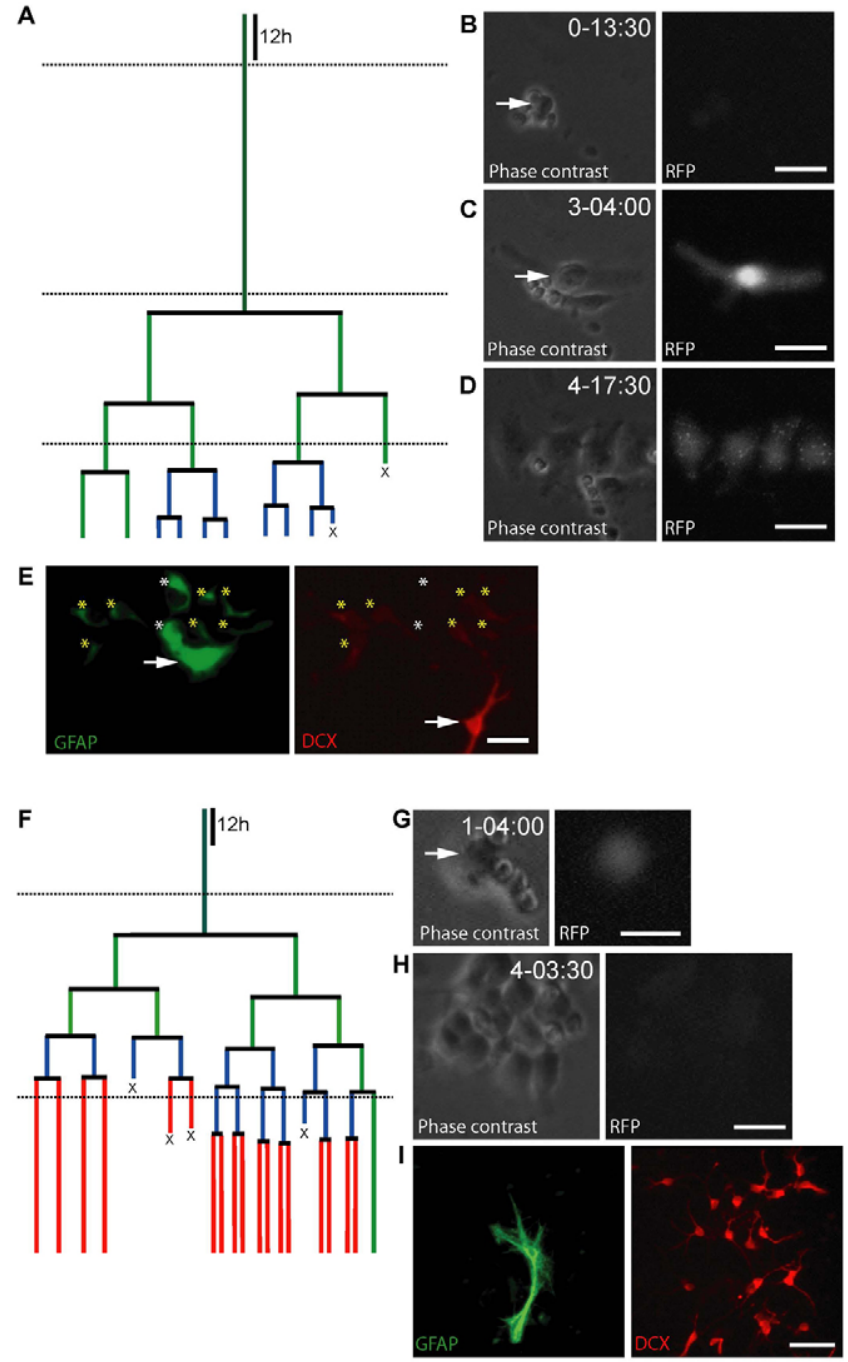

Fig. 6. Astro/radial glial identity of slow-dividing cells. (A) Lineage tree founded by a slow-dividing cell derived from hGFAP-RFP mice (see Fig. 5 for colour key). Note that the cell is initially small and RFPnegative (arrow, B), but progressively grows in size and massively upregulates RFP expression as it approaches cell division (C). (D) Note the marked decrease in RFP expression at the four cell stage of the clone. (E) Post-imaging immunolabelling reveals the astroglial identity of two cells (white asterisks) and the transitional state of sibling TAPs expressing low levels of GFAP and DCX (yellow asterisks). Arrows point to GFAP- and DCX-positive neighbouring cells that do not belong to the clone. (F) Lineage tree from another slow-dividing RFP-positive cell (see Fig. 5 for colour key). Observe the RFP expression in the cell before the first round of cell division (G) and its absence when cells became TAPs or neuroblasts (H). (I) Post-imaging immunolabelling revealed the presence of a single GFAP-positive astroglia alongside many DCX-

positive neurons. $X$ indicates cell death. Dotted horizontal lines indicate the respective time points when pictures were taken. Scale bars: $20 \mu \mathrm{m}$. TAP, transit-amplifying progenitor.

combination with CD133/prominin1 surface expression) (Beckervordersandforth et al., 2010), TAPs (high EGF receptor surface expression) (Pastrana et al., 2009) and neuroblasts (DsRed transgene expression driven from the DCX promoter) (CouillardDespres et al., 2006). Sorted cells were then analysed for their forward scatter as a measure of cell size (Fig. 8; see Fig. S3 in the supplementary material). Figure 8 shows cumulative frequency distributions of the forward scatter for the three different populations. Consistent with our in vitro analysis, we observed a highly significant difference between the size of astroglial NSCs and either TAPs or neuroblasts. These data lend strong support to the notion that the progressive decrease in cell size observed in vitro correlates with a similar decrease during lineage progression in vivo.

\section{EGF and FGF2 greatly enhance symmetric cell division of astroglia-like cells}

We next addressed the question of how these lineage trees become modified by exposure to mitogenic growth factors such as EGF and FGF2. Using adult SEZ cultures derived from hGFAP-RFP mice, several drastic differences could be noted compared with controls following a single addition of both EGF and FGF2 (Fig. 9; see Movies 3, 4 and Fig. S12 in the supplementary material). Whereas in controls the progeny of RFP-positive cells gradually lost RFP expression generating DCX-positive neuroblasts, clones in EGFFGF2-treated cultures retained high levels of hGFAP-RFP expression throughout imaging (Fig. 9). Accordingly, at the end of imaging, the vast majority of cells expressed GFAP consistent with the fact that addition of EGF and FGF2 causes a shift in cell fate favouring astrogliogenesis at the expense of neurogenesis (Doetsch et al., 2002; Kuhn et al., 1997). In addition to modifying cell fate decisions, EGF-FGF2 treatment also affected the mode of cell division. Single cell tracking showed that fast-dividing RFPpositive cells started to divide almost exclusively symmetrically giving rise to fast-dividing astro/radial glia rather than generating RFP-negative TAPs (Fig. 9D', inset). As illustrated by Movie 5 in the supplementary material, slow-dividing RFP-positive astroglia were still encountered in cultures treated with EGF and FGF2 generating fast-dividing RFP-positive progeny, but their lineage progression and mode of division was then severely altered. Thus, EGF-FGF2 treatment seems to act predominantly at the astro/radial glia stage, consistent with the high expression of EGF receptors in the fast-proliferating astroglia (Pastrana et al., 2009). Accordingly, in the presence of EGF and FGF2 the cell cycle length of RFPpositive fast-dividing astroglia was significantly shorter than in the absence of growth factors (without EGF and FGF2: 17.23 \pm 0.6154 hours, $n=34$; with EGF and FGF2: $12.47 \pm 0.5021$ hours, $n=21$; $P<0.001$ determined by Mann-Whitney test; Fig. $\left.9 \mathrm{C}^{\prime}, \mathrm{D}^{\prime}\right)$. Consistent with acceleration of the cell cycle as well as overall enhanced symmetric proliferative cell division in the presence of EGF and FGF2, cultures became confluent by the end of imaging, whereas in controls less than $10 \%$ of the surface area was covered (Fig. 9). Moreover, compared with untreated cultures (Fig. 9C'), in cultures treated with EGF and FGF2, cell growth was markedly increased (Fig. 9D'). Consistent with the symmetric generation of fast-dividing astroglia, no progressive decrease in cell size was observed (Fig. 9D'). Moreover, whereas control cells, without exception, divided while maintaining contact to the poly-D-lysine substrate, following EGF-FGF2 treatment cells lost this requirement and started to divide without adhering to the substrate (see Fig. S12 and Movies 4, 5 in the supplementary material), leading to the formation of typical neurospheres. This indicates that the formation of spheroids in the neurosphere assay (Rietze and Reynolds, 2006) is not simply a consequence of not providing a substrate, but rather an inherent loss of substrate adherence following EGF-FGF2 treatment.

Finally, in sharp contrast to control SEZ cells, which show relatively little migratory behaviour prior to acquiring a neuroblast identity, adult SEZ cells treated with EGF and FGF2 exhibited dramatically increased motility, consistent with reduced stability of 

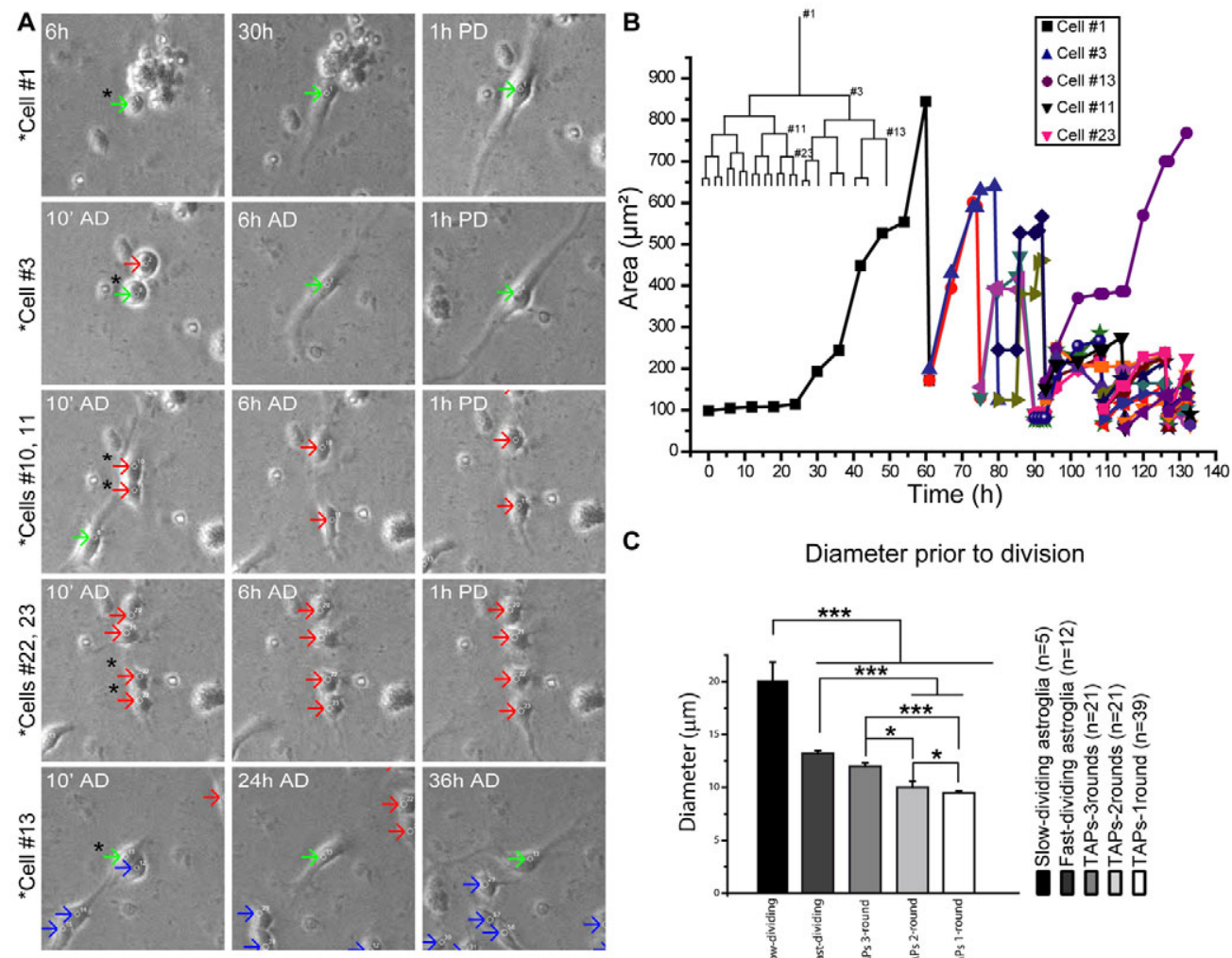

C

Diameter prior to division

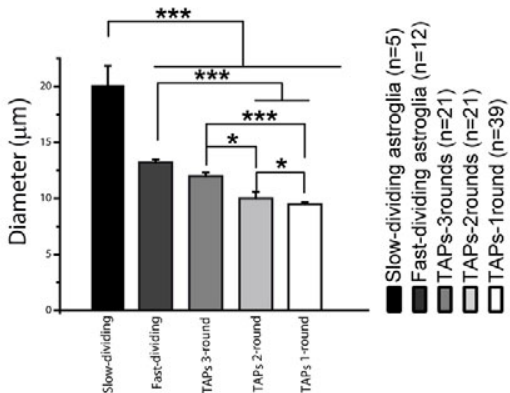

Fig. 7. Cell size and growth pattern correlate with the specific stage of a cell within the lineage of adult NSCs. (A) Cells from the same clone shown in Fig. 4 at different time points after division (AD) and 1 hour prior to division (PD). Green, red and blue arrows indicate indicate an astro/radial glial cell (green), TAPs within the symmetric branch of the lineage tree (red) and TAPs within the asymmetric branch of the lineage tree (blue), respectively. Asterisks indicate the cells highlighted in B. (B) Plotting of the area of cells at different time points and stages within the lineage shown in B. Note that cell growth is markedly reduced as the lineage progresses, with the exception of cell \#13 that retains astro/radial glial features. Inset: Lineage tree showing the position of cells shown in A within the lineage: \#1, slow-dividing astro/radial glia; \#3, fast-dividing astro/radial glia; \#11, TAP undergoing two rounds of cell division; \#23, TAP undergoing one round of cell division; \#13, non-dividing astro/radial glia. (C) Quantification of the cell diameter one frame ( 5 minutes) prior to division (i.e. maximum size) of adult NSCs derived from hGFAP-RFP mice at different stages within the lineage. Error bars indicate s.e.m. ${ }^{*} * P<0.001,{ }^{*} P<0.01,{ }^{*} P<0.05$, ANOVA with Tukey's post-hoc test). Data were obtained from five lineages of slow-dividing astro/radial glia. NSC, neural stem cell; TAP, transit-amplifying progenitor.

contact to the substrate. As can be appreciated from Fig. S12 and Movie 4 in the supplementary material, entire neurospheres migrated and this migration was not due to floating but to active migration over the substrate. Given the dramatic effect of EGF and FGF2 on adult SEZ astroglia, we wondered whether challenge with these growth factors could re-induce cell proliferation in seemingly quiescent astroglial progeny generated by asymmetric cell division (e.g. Fig. 4), thereby allowing assessment of whether these cells had truly become postmitotic or still retained the potential to undergo cell division. To this end, we performed single cell tracking experiments for five consecutive days under control conditions followed by 3 days of EGF-FGF2 treatment. Figure 10 shows an example lineage tree exhibiting an asymmetric cell division (cell \#2) generating neurogenic TAPs and a progenitor (cell \#3) giving rise to cells with astroglial morphology (cells \#4 and 5) (see also Movie 6 in the supplementary material). Of note, both astroglial cells did not undergo further cell division under control conditions, suggesting that they could have become either quiescent or terminally postmitotic. However, following challenge with EGF and FGF2, cell \#4 resumed cell proliferation and remained proliferative until the end of the imaging period, generating a neurosphere-like clonal aggregate. Interestingly, the mode of cell division was highly symmetric (Fig. 10B) and cells exhibited an accelerated cell cycle and markedly enhanced cell growth (Fig. 10C), as previously observed for cultures treated with EGF and FGF2 (Fig. 9D'). Thus, at least some of the astroglial progeny generated by asymmetric cell division are not terminally postmitotic, but retain the potential to resume proliferation.

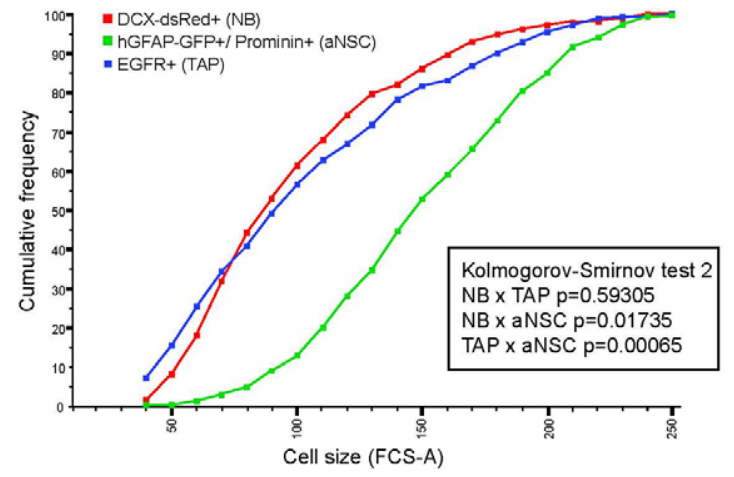

Fig. 8. Cell size decreases significantly (Kolmogorov-Smirnov test) from astroglial stem cells to TAPs or neuroblasts in vivo. The cumulative frequency distributions of forward scatter (FCS-A) as a measure for cell size for freshly isolated astroglial stem cells (hGFAPGFP+/prominin+), TAPs (EGFR+) and neuroblasts (DCX-dsRed) following fluorescence-activated cell sorting. TAP, transit-amplifying progenitor. 
A

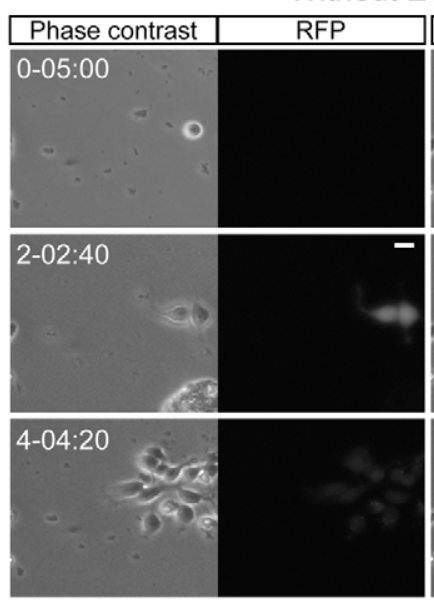

C

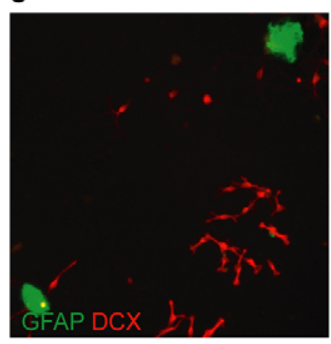

C'
Without EGF/FGF2
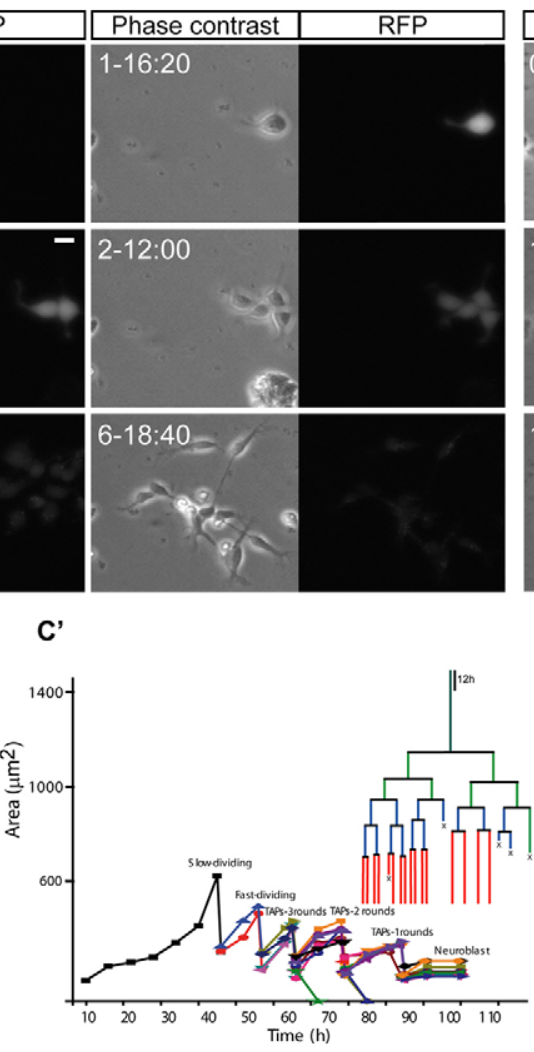

B
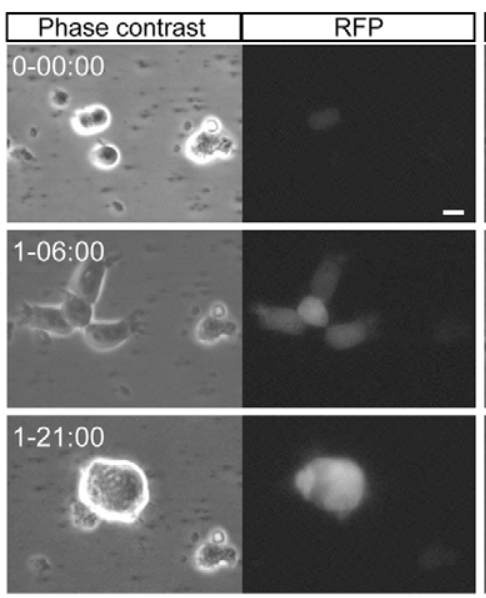

D

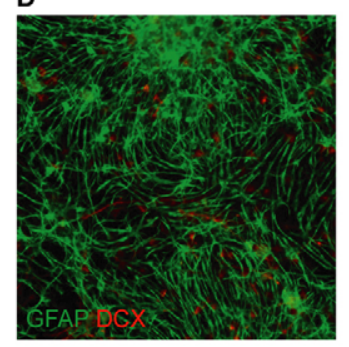

D'
With EGF/FGF2
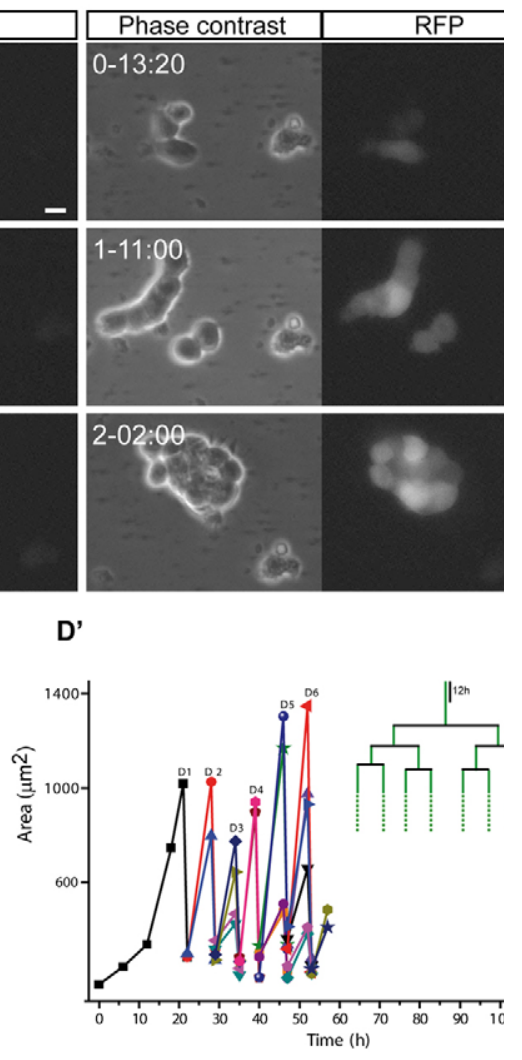

Fig. 9. Treatment of adult SEZ cells with EGF and FGF2 massively alters the behaviour of adult SEZ astroglia. (A,B) Time-lapse sequences (day-hour:minute) of SEZ cells prepared from hGFAP-RFP mice cultured without growth factors (A) or in the presence of EGF and FGF2 (B). Note that the progeny of the clone-founding cell retains high levels of RFP expression and eventually form a neurosphere-like structure. Cells continued to divide until forming a monolayer composed predominantly of astrocytes (as shown in D). (C,D) Micrographs of SEZ cells cultured for 7 days in the absence (C) or presence (D) of EGF and FGF2 and immunolabelled for GFAP and DCX. Box in C shows the DCX staining of cells depicted in A (at 608:00). ( $\left.\mathbf{C}^{\prime}\right)$ Progressive decrease in cell area along the lineage progression of the clone depicted in A. Inset: Lineage tree of the cell shown in $A$ (see Fig. 5 for colour key). ( $\left.\mathbf{D}^{\prime}\right)$ Growth pattern of a clone exposed to EGF and FGF2. Note that cell growth is markedly enhanced compared with control conditions, and the cell cycle is markedly accelerated. The growth pattern could not be followed beyond the sixth division. Inset: Lineage tree of one of the cells shown in B (see Fig. 5 for colour key). Scale bars: $20 \mu \mathrm{m}$. SEZ, subependymal zone.

\section{DISCUSSION}

Here, we describe a cell culture preparation of the adult SEZ that allows examination of the behaviour of adult NSCs and their progeny in isolation from their niche. Several lines of evidence support the notion that the behaviour of neural stem and precursor cells observed in this preparation closely resembles their behaviour in vivo, with the notable difference that it takes place in the absence of a stem cell niche. First, the majority of progeny in this culture is neuronal and mostly of GABAergic identity with only a minor glutamatergic lineage, as observed in vivo (Brill et al., 2008, Brill et al., 2009). Second, NSCs have astroglial characteristics, are characterised by a slow cell cycle, and lineage progression from NSCs to neuroblasts occurs through the same basic sequence as proposed on a population basis (Kriegstein and Alvarez-Buylla, 2009). Third, the shuttling between a slow- and fast-dividing state observed here directly has been suggested to occur in the other neurogenic region of the adult brain, the dentate gyrus (Lugert et al., 2010), consistent with the possibility that such shuttling is a conserved feature of adult NSCs. Fourth, the decrease in cell size during lineage progression from NSC to neuroblasts is in accordance with the differences in cell size between freshly isolated astroglial stem cells and TAPs or neuroblasts (see Fig. 8). Finally, the massive astrogliogenesis and altered migratory behaviour (Fig. 9) that occurs as a result of EGF-FGF2 treatment on adult NSCs corresponds well with similar observations in vivo (Doetsch et al., 2002; Kuhn et al., 1997).

Most importantly, however, this culture system permits the continuous observation of the transitions between stem and progenitor cell types and their neuronal progeny. Our data show unambiguously that, following isolation from their niche, adult astro/radial glia retain stem cell properties, such as their rather slow cell cycle and their ability to generate neuroblasts through a highly ordered sequence of intermediates. Moreover, it provides for the first time direct evidence for asymmetric cell division within the lineage of adult NSCs, but with the surprising twist that it is not the slow-dividing astro/radial NSC itself but its immediate fastdividing astroglial progeny that undergoes asymmetric cell division. Taken together, these data demonstrate a central cellintrinsic component in adult NSC lineage progression. The fact that lineage progression from NSCs to neuroblasts occurs in such highly ordered fashion as well as the reproducible position of asymmetric cell division within lineage progression, is consistent with the notion that a considerable part of the programme unfolded by NSCs can be enacted in the absence of a niche, as has been 
A

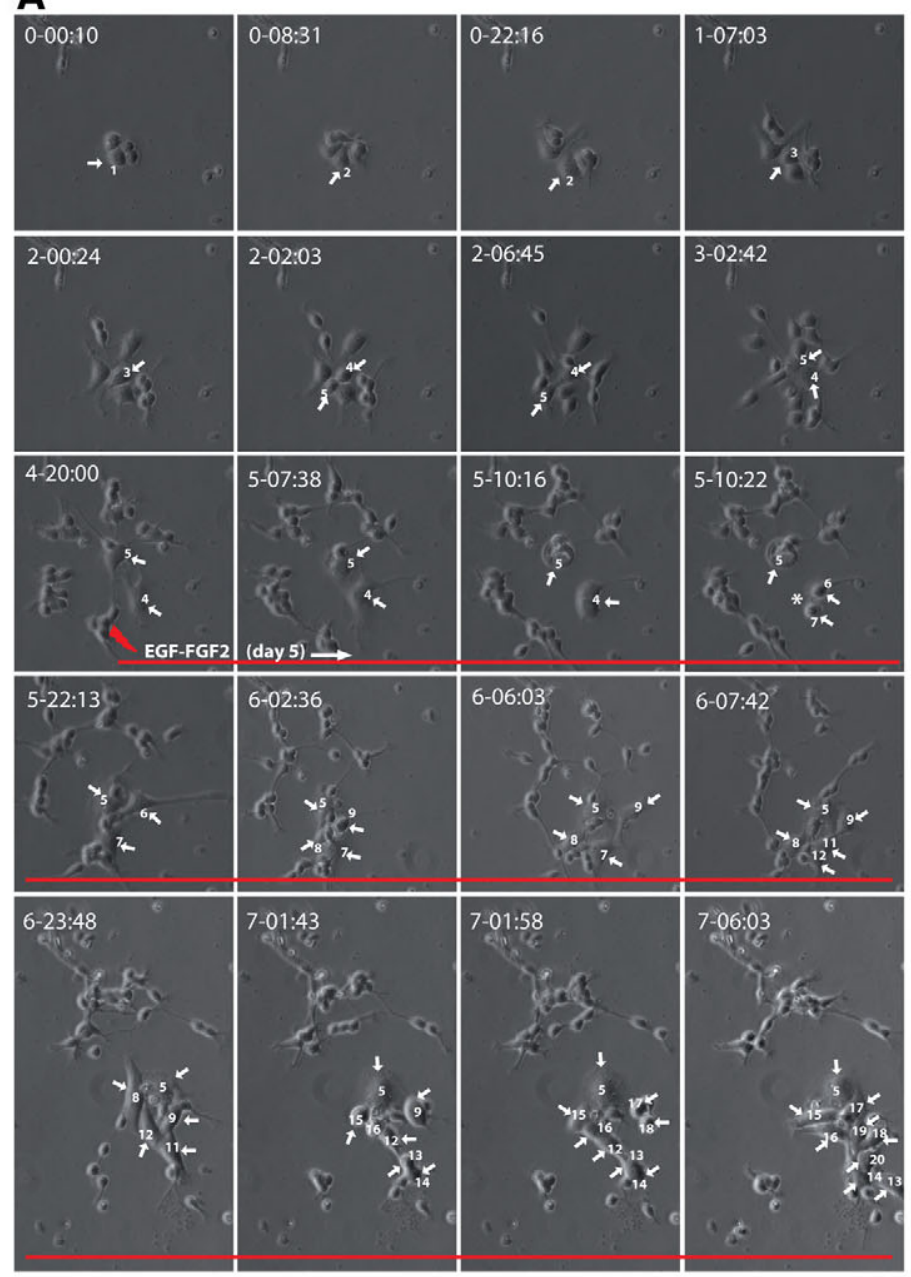

B
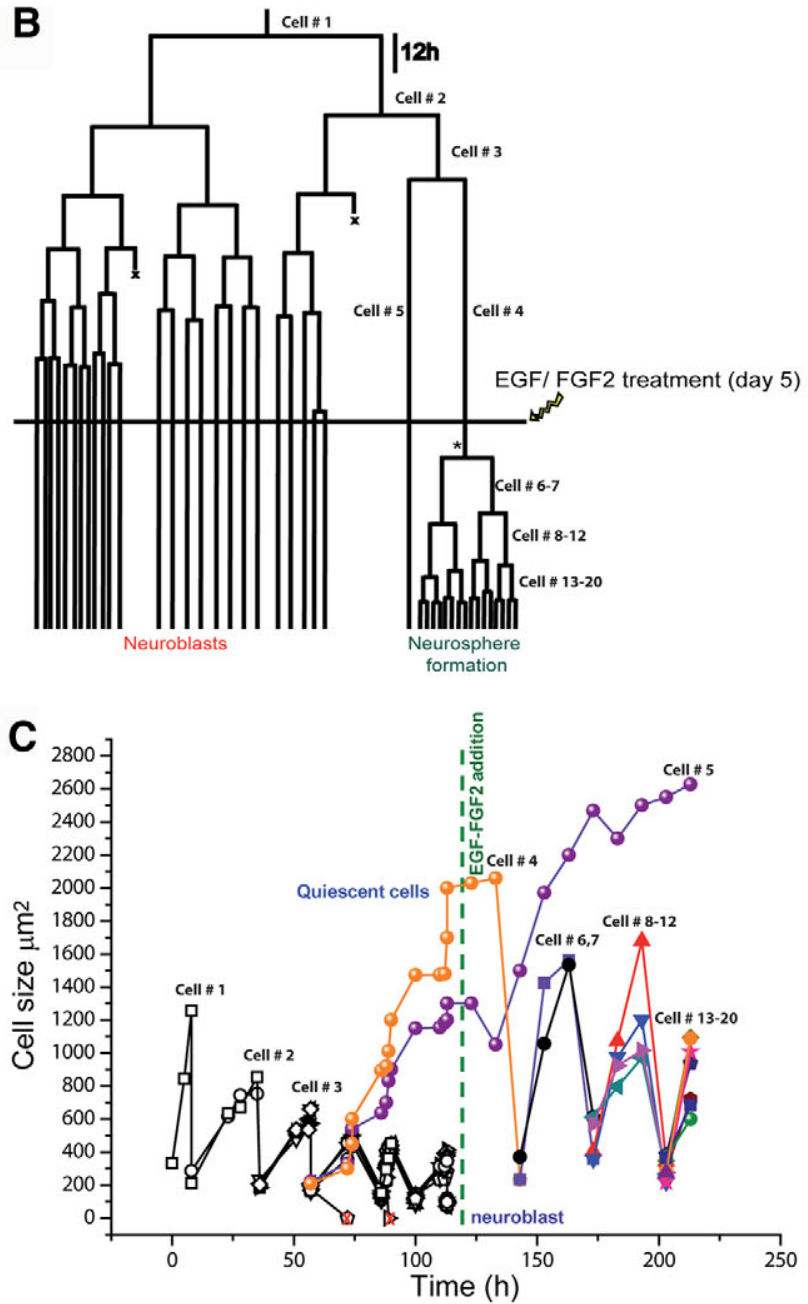

Fig. 10. Quiescent astroglial progeny generated by asymmetric cell division can re-enter the cell cycle after challenge with EGF and FGF2. (A) Phase contrast images of a clone show a cell (cell \#1) giving rise to a symmetric neurogenic branch and a branch exhibiting asymmetric cell division (cell \#2). Whereas one of the daughter cells of cell \#2 generates neuroblasts, the other (cell \#3) gives rise to two astroglial cells that become seemingly quiescent. One of these cells (cell \#4) re-enters the cell cycle after stimulation with EGF and FGF2 (red line, day 5 onwards) generating a neurospherelike clonal aggregate (comprising cells \#6-20) demonstrating that it has not become permanently postmitotic. White asterisk indicates the moment prior to cell division. (B) Lineage tree of the clone depicted in A. Note that treatment with EGF and FGF2 induces proliferation of quiescent astroglial progeny (black asterisk) but does not affect postmitotic neuroblasts. (C) Cell size analysis along lineage progression.

previously demonstrated for isolated cortical stem and precursor cells from the developing neocortex (Shen et al., 2006; Temple, 1989). Obviously, these data do not imply that the niche plays no important modulatory role in regulating the behaviour of NSCs of different origins, but only indicates that the basic sequence of lineage progression can unfold in its absence.

Amongst the clones containing astro/radial glia-like cells, single cell tracking experiments also revealed highly reproducible sequences of cell division and lineage progression (Fig. 11): (1) slow-dividing astro/radial glia divide symmetrically once or twice generating fast-dividing astro/radial glia; (2) fast-dividing astro/radial glia divide either symmetrically generating TAPs or asymmetrically generating an astro/radial glial cell and TAPs (Fig. 11, flash arrow); (3) fast-dividing cells comprising fast-proliferating astro/radial glia and TAPs divide symmetrically up to five times.

Interestingly, two types of astro/radial glial cells have been described in the neurogenic niches. Both in the SEZ and the subgranular zone (SGZ) of the dentate gyrus, slow-dividing astro/radial glial cells have been considered to be the bona fide NSCs (exhibiting a radial morphology in the SGZ and SEZ), whereas a faster dividing cell with astroglial features (with a horizontal morphology in the SGZ) has been considered to be 'activated' (Doetsch et al., 2002; Mirzadeh et al., 2008; Pastrana et al., 2009) but, at least in the SGZ, also exhibits NSC properties (Suh et al., 2007). However, the lineage relationship between these cell types remained elusive. Here, we provide compelling evidence that fast-dividing astro/radial glia in the SEZ are indeed the direct progeny of slow-dividing NSCs.

In their asymmetric divisions, fast-dividing astro/radial glia generate TAPs that in turn give rise in a very stereotyped manner to neuroblasts, while also producing a cell with astro/radial glia features that, after few rounds, exits the fast division mode. It was impossible to determine within the imaging time whether astroglial progeny generated by asymmetric cell division are permanently postmitotic or revert to a slow-dividing astro/radial glial phenotype as observed at the beginning of the lineage. However, by 


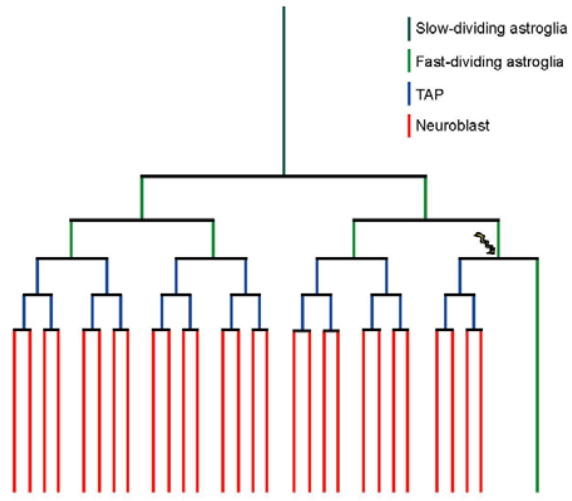

Fig. 11. Model for the lineage progression of adult SEZ NSCs. Slowdividing astroglia NSCs divide symmetrically generating two fast-dividing astroglia, which in turn divide symmetrically to generate four fast-dividing astroglia. Although most of these cells continue to divide symmetrically thus giving rise to neuron-producing TAPs, typically one of them undergoes asymmetric cell division (yellow flash arrow), generating neuron-producing TAPs and one or a few astroglial cells. NSC, neural stem cell; SEZ, subependymal zone; TAP, transit-amplifying progenitor.

challenging cultures with the growth factors EGF and FGF2 following 5 days of imaging under control conditions we could observe that these astroglial progeny can resume proliferation giving rise to neurosphere-like clonal aggregates (Fig. 10; see Movie 6 in the supplementary material). These data indicate that at least a subpopulation of these astroglial progeny has not become terminally postmitotic, but retains the potential to proliferate, consistent with the possibility that these seemingly quiescent astroglial cells retain NSC characteristics. Thus, our results suggest that (1) astro/radial glia NSCs might shuttle between fast and slow proliferation and (2) the phenomenon of NSC self-renewal might require both symmetric division of the slow-dividing cells and asymmetric division of the fast-dividing astro/radial glia cells.

Single cell tracking also permitted us to directly observe a unidirectional progression from slow-dividing astro/radial glia to fast-dividing astro/radial glia, TAPs and neuroblasts. In no single case did we observe TAPs giving rise to astroglia or any nonneuronal cell type, suggesting that the generation of TAPs from activated astroglia could be a crucial step in the commitment of cells towards a neuronal phenotype.

Last but not least, the behaviour exhibited by NSCs and their progeny in the absence of exogenously added mitogenic growth factors is highly suggestive of cell-intrinsic or pre-programmed mechanisms determining the sequence of lineage progression. In particular, the substantial growth of slow-dividing cells prior to cell division along with the subsequent reduction in cell size with further divisions suggests a cell-intrinsic programme that is initiated already within the slow-dividing cell and then unfolds itself during the subsequent divisions. Intriguingly, cells retaining astro/radial glial identity throughout the lineage differ from their other siblings in their growth pattern and instead resemble their slow-dividing ancestors of astro/radial glial identity (Figs 7, 10). Thus, our observations of mammalian adult NSC divisions might suggest that factors regulating cell size contribute to the decision for self-renewal or lineage progression as well as the number of subsequent cell divisions of the progeny. Similar mechanisms have been shown to operate in Drosophila neuroblasts and have been implicated in ovary cancer formation (Neumuller et al., 2008).
Interestingly, EGF and FGF treatment also seems to affect this aspect, as cells, despite the accelerated cell cycle, exhibit an enhanced growth rate, which is of notable difference to the also fast-dividing TAPs that decrease in size with each division.

The upregulation of RFP expression in the slow-dividing astro/radial glia prior to cell division highlights dynamic changes in the transcriptional profile of slow-dividing NSCs when entering mitosis, which might be a prerequisite for the massive cell growth of slow-dividing cells that occurs in parallel. The striking correlation between cell size and the number of subsequent divisions (Fig. 7; see Fig. S2 in the supplementary material) prompts the suggestion that one potential key difference between stem cells undergoing a theoretically unlimited number of cell divisions and the TAPs with rather limited cell divisions (three to five divisions in this system) is indeed cell growth, with the inability to sufficiently increase cell size between individual divisions as a key mechanism to restrict the number of cell cycles to be completed. The fact that cultures treated with EGF and FGF2, which predominantly generate astroglial progeny, exhibit a markedly distinct pattern of cell growth compared with control cultures (Fig. 9) lends further support to the notion of mechanisms regulating cell growth also affecting cell fate decisions. Notably, although under control conditions larger cell size goes hand in hand with a longer cell cycle (slow dividing cells), cell cycle length and growth rate are inversely modified by mitogenic growth factors indicating that growth is not simply a passive consequence of the duration of the cell cycle. Finally, the conspicuous growth pattern and changes in cell size together with cell cycle length and the dynamic regulation of hGFAP-driven RFP expression could be used to provide crucial online information regarding the identity of a given cell that could be utilised for the computational prediction of neural progenitor fate (Cohen et al., 2010; Schroeder, 2010).

Whereas NSCs and their progeny exhibit a highly stereotypic behaviour in the absence of growth factors, treatment with EGF and FGF2 results in massive alterations of their rather tightly regulated behaviour: increased generation of fast-dividing astroglia by symmetric proliferative division at the expense of neuronproducing TAPs, acceleration of the cell cycle, increased cell growth, enhanced astrogliogenesis, partial loss of substrate contact (resulting in the formation of spheroids), and aberrant migration, all properties highly reminiscent of glioma cells (D'Abaco and Kaye, 2007). Thus, live imaging demonstrated that the massive astrogliogenesis (instead of neurogenesis) observed upon FGF2 or EGF infusion in vivo or after growth factor treatment in vitro is due to an instructive effect of EGF and FGF2 affecting both the mode of cell division and the fate specification of fast-proliferating astro/radial glial cells.

In summary, the live imaging system of primary cultures of adult SEZ cells provides the first model system to study the molecular and cellular mechanisms governing the transition from slow- to fast-dividing adult NSCs, the process and regulation of asymmetric cell division, and how alterations of the mode of cell division might affect fate decisions in this crucial lineage.

\section{Acknowledgements}

We thank Drs Chris Doe, Ben Simons and Marco Canossa for critical reading of and helpful discussions on the manuscript, Dr Frank Kirchhoff (MPI for Experimental Medicine, Goettingen, Germany) for generously providing us with the hGFAP-RFP mouse line and Jorge Ramirez Franco for help with the ImageJ software. We are also very grateful to Tatiana Simon-Ebert and Joanna Zaißerer for their excellent technical assistance. This work was supported by grants from the Deutsche Forschungsgemeinschaft, including SFB 596, SPP1356, the LMU Excellent Program and the Excellence Cluster CIPS, the 
BMBF, EUTRACC and the Bavarian State Ministry of Sciences, Research and the Arts to B.B., T.S. and M.G. M.R.C. was supported by an Alexander-vonHumboldt postdoctoral fellowship

\section{Competing interests statement}

The authors declare no competing financial interests.

\section{Supplementary material}

Supplementary material for this article is available at

http://dev.biologists.org/lookup/suppl/doi:10.1242/dev.061663/-/DC1

\section{References}

Alonso, M., Ortega-Perez, I., Grubb, M. S., Bourgeois, J. P., Charneau, P. and Lledo, P. M. (2008). Turning astrocytes from the rostral migratory stream into neurons: a role for the olfactory sensory organ. J. Neurosci. 28, 11089-11102.

Beckervordersandforth, R., Tripathi, P., Ninkovic, J., Bayam, E., Lepier, A., Stempfhuber, B., Kirchhoff, F., Hirrlinger, J., Haslinger, A., Lie, D. C. et al. (2010). In vivo fate mapping and expression analysis reveals molecular hallmarks of prospectively isolated adult neural stem cells. Cell Stem Cell 7, 744-758.

Brill, M. S., Snapyan, M., Wohlfrom, H., Ninkovic, J., Jawerka, M., Mastick, G. S., Ashery-Padan, R., Saghatelyan, A., Berninger, B. and Gotz, M. (2008). A dlx2- and pax6-dependent transcriptional code for periglomerular neuron specification in the adult olfactory bulb. J. Neurosci. 28, 6439-6452.

Brill, M. S., Ninkovic, J., Winpenny, E., Hodge, R. D., Ozen, I., Yang, R., Lepier, A., Gascon, S., Erdelyi, F., Szabo, G. et al. (2009). Adult generation of glutamatergic olfactory bulb interneurons. Nat. Neurosci. 12, 1524-1533.

Cohen, A. R., Gomes, F. L., Roysam, B. and Cayouette, M. (2010). Computational prediction of neural progenitor cell fates. Nat. Methods 7, 213-218.

Colak, D., Mori, T., Brill, M. S., Pfeifer, A., Falk, S., Deng, C., Monteiro, R. Mummery, C., Sommer, L. and Gotz, M. (2008). Adult neurogenesis require Smad4-mediated bone morphogenic protein signaling in stem cells. J. Neurosci. 28, 434-446

Costa, M. R., Wen, G., Lepier, A., Schroeder, T. and Gotz, M. (2008). Par-complex proteins promote proliferative progenitor divisions in the developing mouse cerebral cortex. Development 135, 11-22.

Couillard-Despres, S., Winner, B., Karl, C., Lindemann, G., Schmid, P., Aigner,

R., Laemke, J., Bogdahn, U., Winkler, J., Bischofberger, J. et al. (2006). Targeted transgene expression in neuronal precursors: watching young neurons in the old brain. Eur. J. Neurosci. 24, 1535-1545.

D'Abaco, G. M. and Kaye, A. H. (2007). Integrins: molecular determinants of glioma invasion. J. Clin. Neurosci. 14, 1041-1048.

Doetsch, F., Petreanu, L., Caille, I., Garcia-Verdugo, J. M. and Alvarez-Buylla, A. (2002). EGF converts transit-amplifying neurogenic precursors in the adult brain into multipotent stem cells. Neuron 36, 1021-1034.

Eilken, H. M., Nishikawa, S. and Schroeder, T. (2009). Continuous single-cell imaging of blood generation from haemogenic endothelium. Nature 457, 896900 .

Hack, M. A., Sugimori, M., Lundberg, C., Nakafuku, M. and Gotz, M. (2004). Regionalization and fate specification in neurospheres: the role of Olig2 and Pax6. Mol. Cell. Neurosci. 25, 664-678.

Hack, M. A., Saghatelyan, A., de Chevigny, A., Pfeifer, A., Ashery-Padan, R., Lledo, P. M. and Gotz, M. (2005). Neuronal fate determinants of adult olfactory bulb neurogenesis. Nat. Neurosci. 8, 865-872

Heinrich, C., Blum, R., Gascon, S., Masserdotti, G., Tripathi, P., Sanchez, R., Tiedt, S., Schroeder, T., Gotz, M. and Berninger, B. (2010). Directing astroglia from the cerebral cortex into subtype specific functional neurons. PLoS Biol. $\mathbf{8}$ e1000373.

Hirrlinger, P. G., Scheller, A., Braun, C., Quintela-Schneider, M., Fuss, B., Hirrlinger, J. and Kirchhoff, F. (2005). Expression of reef coral fluorescent proteins in the central nervous system of transgenic mice. Mol. Cell. Neurosci. 30 , 291-303

Johansson, C. B., Svensson, M., Wallstedt, L., Janson, A. M. and Frisen, J. (1999). Neural stem cells in the adult human brain. Exp. Cell Res. 253, 733-736.

Kriegstein, A. and Alvarez-Buylla, A. (2009). The glial nature of embryonic and adult neural stem cells. Annu. Rev. Neurosci. 32, 149-184.

Kuhn, H. G., Winkler, J., Kempermann, G., Thal, L. J. and Gage, F. H. (1997). Epidermal growth factor and fibroblast growth factor-2 have different effects on neural progenitors in the adult rat brain. J. Neurosci. 17, 5820-5829.
Laywell, E. D., Rakic, P., Kukekov, V. G., Holland, E. C. and Steindler, D. A. (2000). Identification of a multipotent astrocytic stem cell in the immature and adult mouse brain. Proc. Natl. Acad. Sci. USA 97, 13883-13888.

Lim, D. A. and Alvarez-Buylla, A. (1999). Interaction between astrocytes and adult subventricular zone precursors stimulates neurogenesis. Proc. Natl. Acad. Sci. USA 96, 7526-7531.

Lugert, S., Basak, O., Knuckles, P., Haussler, U., Fabel, K., Gotz, M., Haas, C. A., Kempermann, G., Taylor, V. and Giachino, C. (2010). Quiescent and active hippocampal neural stem cells with distinct morphologies respond selectively to physiological and pathological stimuli and aging. Cell Stem Cell 6, 445-456.

Merkle, F. T., Mirzadeh, Z. and Alvarez-Buylla, A. (2007). Mosaic organization of neural stem cells in the adult brain. Science 317, 381-384.

Mira, H., Andreu, Z., Suh, H., Lie, D. C., Jessberger, S., Consiglio, A., San Emeterio, J., Hortiguela, R., Marques-Torrejon, M. A., Nakashima, K. et al. (2010). Signaling through BMPR-IA regulates quiescence and long-term activity of neural stem cells in the adult hippocampus. Cell Stem Cell 7, 78-89.

Mirzadeh, Z., Merkle, F. T., Soriano-Navarro, M., Garcia-Verdugo, J. M. and Alvarez-Buylla, A. (2008). Neural stem cells confer unique pinwheel architecture to the ventricular surface in neurogenic regions of the adult brain. Cell Stem Cell $\mathbf{3}$ 265-278.

Neumuller, R. A., Betschinger, J., Fischer, A., Bushati, N., Poernbacher, I. Mechtler, K., Cohen, S. M. and Knoblich, J. A. (2008). Mei-P26 regulates microRNAs and cell growth in the Drosophila ovarian stem cell lineage. Nature $\mathbf{4 5 4}$ 241-245.

Nolte, C., Matyash, M., Pivneva, T., Schipke, C. G., Ohlemeyer, C., Hanisch, U. K., Kirchhoff, F. and Kettenmann, H. (2001). GFAP promoter-controlled EGFPexpressing transgenic mice: a tool to visualize astrocytes and astrogliosis in living brain tissue. Glia 33, 72-86.

Pastrana, E., Cheng, L. C. and Doetsch, F. (2009). Simultaneous prospective purification of adult subventricular zone neural stem cells and their progeny. Proc Natl. Acad. Sci. USA 106, 6387-6392.

Qian, X., Goderie, S. K., Shen, Q., Stern, J. H. and Temple, S. (1998). Intrinsic programs of patterned cell lineages in isolated vertebrate CNS ventricular zone cells. Development 125, 3143-3152.

Qian, X., Shen, Q., Goderie, S. K., He, W., Capela, A., Davis, A. A. and Temple, S. (2000). Timing of CNS cell generation: a programmed sequence of neuron and glial cell production from isolated murine cortical stem cells. Neuron 28, 69-80.

Ravin, R., Hoeppner, D. J., Munno, D. M., Carmel, L., Sullivan, J., Levitt, D. L., Miller, J. L., Athaide, C., Panchision, D. M. and McKay, R. D. (2008). Potency and fate specification in CNS stem cell populations in vitro. Cell Stem Cell 3, 670680

Reynolds, B. A. and Weiss, S. (1992). Generation of neurons and astrocytes from isolated cells of the adult mammalian central nervous system. Science $\mathbf{2 5 5}$, 17071710.

Reynolds, B. A. and Rietze, R. L. (2005). Neural stem cells and neurospheres-reevaluating the relationship. Nat. Methods 2, 333-336.

Rieger, M. A. and Schroeder, T. (2009). Analyzing cell fate control by cytokines through continuous single cell biochemistry. J. Cell. Biochem. 108, 343-352.

Rietze, R. L. and Reynolds, B. A. (2006). Neural stem cell isolation and characterization. Methods Enzymol. 419, 3-23.

Schroeder, T. (2008). Imaging stem-cell-driven regeneration in mammals. Nature 453, 345-351

Schroeder, T. (2010). The electronic crystal ball: predicting cell fate from time-lapse data. Nat. Methods 7, 190-191.

Shen, Q., Wang, Y., Dimos, J. T., Fasano, C. A., Phoenix, T. N., Lemischka, I. R., Ivanova, N. B., Stifani, S., Morrisey, E. E. and Temple, S. (2006). The timing of cortical neurogenesis is encoded within lineages of individual progenitor cells. Nat. Neurosci. 9, 743-751

Suh, H., Consiglio, A., Ray, J., Sawai, T., D'Amour, K. A. and Gage, F. H. (2007) In vivo fate analysis reveals the multipotent and self-renewal capacities of Sox2+ neural stem cells in the adult hippocampus. Cell Stem Cell 1, 515-528.

Tamamaki, N., Yanagawa, Y., Tomioka, R., Miyazaki, J., Obata, K. and Kaneko, T. (2003). Green fluorescent protein expression and colocalization with calretinin, parvalbumin, and somatostatin in the GAD67-GFP knock-in mouse. J. Comp. Neurol. 467, 60-79.

Temple, S. (1989). Division and differentiation of isolated CNS blast cells in microculture. Nature 340, 471-473.

Zhao, C., Deng, W. and Gage, F. H. (2008). Mechanisms and functional implications of adult neurogenesis. Cell 132, 645-660. 